starts operation in 1993-4. The next meeting of the Swiss user community in February 1991 will consider once more the interest in SLS. Establishing a larger constituency of critical mass will depend on a number of local factors, notably the outcome of a proposal to build a $2 \mathrm{~nm}$ source for fabricating micromechanical devices using the new LIGA process at Karlsruhe in Southern Germany; the possibility that a new version of the French Super ARCO machine will be built in Strasbourg; and the likelihood that the Austrian community will have decided to participate in Elettra.

Slightly more removed is the proposed BESSY II source in Berlin (a government official announced on 20 November at the ceremony marking the merger of the German and former DDR physical societies that BESSY II would now be promoted after a considerable delay). A source (DELTA) tailored for testing insertion devices is being built in Dortmund. It will probably be converted eventually to a conventional source available to users. Finally, the Daresbury Laboratory in the UK has proposed a soft X-ray source similar to BESSY II.

Several major synchrotron facilities are under construction further afield (ESRF, Grenoble, France; APS, Argonne, USA; ALS, Berkeley; STEP-8, Osaka-Kobe, Japan) - hence the PSI's proposal for a third generation source offering unique performance features that are not easily available to a large regional community.

\section{Science for Peace Prize}

Professor A. Zichichi, Director of the Erice Centre, has announced that the first Ettore Majorana-Erice-Scienza per la Pace Prize has been awarded, in three equal parts, to Professors E. Teller and V.F. Weisskopf and in memory of the late physicists P.A.M. Dirac, P.L. Kapitza and A.D. Sakharov (whose portion was received by Mrs M. Dirac, Mrs A. Kapitza and Mrs E. Bonner Sakharova). The award ceremony took place at the Ettore Majorana Centre, Sicily on 27 November.

Applied Physics, Physics in Industry Europhysics Industrial Workshops EIW-4, ion beams, 18 EIW-5, ceramic design, 214 EIW-7, surface science, 85 ,

Report, 179
Associate Members

Akzo, joins, 107

Heraeus-Stiftung, contribution, 59

Institut Laue-Langevin, 58, 89

News from Associate Members, 58

Paul Scherrer Institute, 58, 218

World Laboratory, joins, 58

Astronomy and Astrophysics

Barred galaxies, 3

Black hole, stars, 143

European Astronomical Society, 216

FOC, 139

Heterodyne spectroscopy, 195

Hubble telescope, 85

SOFIA, 119, 120

Atomic and Molecular Physics

Ion traps, atomic clocks, 31

Ceramic components, design, 214

Chaotic phenomena, systems, 7

Condensed matter

Magnetism, layered structures, 103

Phase conjugation, mid-IR, 11

Polymers, nonlinear optical, 83

Who's Who, 76

Conference Listings, 46, 183

Databases, numeric, 217

Education

Software, 10

Courses, UK, 107

Europhysics Notes, 179, 216

European Community

Conference support, 18

Funding, 18, 218

Researchers' Europe, 99

Science Plan, assessed, 152

European Physical Society

Associate Members, 58, 107, 130

Collaborating Societies, 129

Council delegates, 117, 127, 153

Council report, 95, 100

Division, Group and Section Boards, 131

East-west report, 137

EPS-8 General Conference

Best poster, 175

Closing Ceremony, 174

\section{Authors}

Altarelli M., 43-45 Andreoni C., 147-151 Anselmetti D., 72-73

Appert K., 156

Bellotti E., 123-126

Benedek G., 71

Besenbacher F., 68-71

Burton W.B., 154
Cyrot-Lackmann F., 151 Di Lella L., 203-207 Dorubantu I.A., 38 Eades J., 27-30 Eggen $H_{\text {., }}$ 157-160 Eliel E., 157 Fierz M., 178

Frois B., 213-214

Güntherodt H.J., 72-73

Hacinliyan A., 7-10

\title{
Index, Vol. 21, 1990
}

\section{Principal Subjects}

Official Reception, 173

Opening, Casimir, H.G.B., 163

Parallel Symposia, 168

Plenary Sessions, 164

Plenary Sessions, 164
Programme, 60, 163

Programme,
report, 179

Europhysics News, Board, 115, 117

Executive Committee, 127

General Meeting

Agenda, 135

announcement, 30

report, 176

Invests for growth, 17

Meetings, 134

Member Organizations, 128

New members, 34, 78, 179

Organized Conferences (1990), 135

President's report, 75

Publications, 135

Recognized Journals, 135

Research facilities, 10

Secretariats, 136, 220

Travel grants, 15

European Research Conferences, 135

Flying Circus, 175

Fusion

AEA Technology, 16

Beryllium, benefits, 14

Fusion energy, 218

Hewlett-Packard Europhysics Prize

Call for Nominations, 76

winners, 18, 72

High Energy Physics

Antiproton, 27

Dirac P., impact, 116

ECFA survey, 93

Gran Sasso, 123

LHC, physics, 210

Quarks, discovery, 208

Superconducting magnets, 90

Top quark, 203

Letters to the Editor, 38, 119, 156, 178

Mathematical physics, institute, Cambridge, 98

Neural networks, physics, 108

Nuclear Physics

Address Booklet, 180

Berlin reactor, 15

Hadronic, with electrons, 213

ILL reactor, 89

NUPECC, 140,180

Planning, Europe, 111
Superdeformed bands, 86

Theory Centre, 113

Obituaries

Amaldi E., 26

Bell J.S., 212

Harbeke G., 71

Lüscher E., 151

Van Hove L., 178

Oort J.H., 90th birthday, 154

Physics and Development

IPPS, Sweden, 118

Physics and Society

Heat store, 76

Seminar, Bad Honnef, energy, 94

Plasma Physics

Division Board, 156

Prizes

Gentner-Kastler, 1990 winner, 59

Hewlett-Packard, 1990 winners, 18, 172

Holweck, 1990 winner, 79

Max Born, 1990 winner, 79

Nobel Prize, physics

1989 winners, 33

1990 winners, 200, 209

Scienza per la Pace, winners, 219

Quantum Electronics, Board, 117

Scanning tunneling microscopy

Imaging theories, 63

Instrumentation, design, 72

Microscopes, 74

Studies, 68

Scherrer P., 100th anniversary, 107

Schools, listings, 54, 191

Societies

Asia Pacific, 180

Balkan, 180

Czechoslovakia, journals, 180

France

French physics, 16

journals, 180

German, unity, 78, 180

IOP, Environmental physics, 117

Japan, members' rates, 180

The Netherlands, physics, 157

USSR, 117

Spain, research spending, 146

Synchrotron radiation

ESRF, Grenoble, 43

Swiss Light Source, 218

Teraflops, 156
Hilf E.R., 38

Jacob M., 178, 208-210, 212 Merz W.J., 107

Janot C., 23-26

Katgert-Merkelijn J.K., 154 Möhlmann G.R., 83-85

Kinzel W., 108-110

Kovalev V.I. 11-14

Kreigreis A., 119

Lucas A.A., 63-67

Luminet J.-P., 143-146

Mackintosh A.R., 76
Mortensen K., 68-71

Nazarewicz W., 86-89

Nieuwenhuizen T., 158

Nitzschmann B.G.J., 38

Pals J.A., 18

Pascovi G., 38
Perin R., 90

Pescia D., 103-107

Pfenniger D., 3-6

Schleich E., 31-33 Veringa M., 214-215

Schreuder H.E., 160

Shaw E.N., 115

Sick I., 111-113

Stacey D.N., 156
Walther $\mathrm{H}_{\text {., }} 31-33$

Wesendanger R., 72-73

Windsor C.G., 147-151

Zichichi A., 116-117 